\title{
AVALIAÇÃO DO POTENCIAL DE USO DE ESPÉCIES DE PÍNUS TROPICAIS E EUCALIPTO NA PRODUÇÃO DE PAINÉIS COMPENSADOS UREICOS
}

\author{
Setsuo Iwakiri ${ }^{1}$, Felipe Gustavo Sanches ${ }^{2}$, Daniele Cristina Potulski², Janice Bernardo da Silva ${ }^{3}$, \\ Mário de Andrade ${ }^{4}$, Raquel Marchesan ${ }^{5}$
}

${ }^{1}$ Eng. Florestal, Dr., Depto. de Engenharia e Tecnologia Florestal, UFPR, Curitiba, PR, Brasil - setsuo@ufpr.br ${ }^{2}$ Eng. Ind. Madeireiro, Mestrando em Engenharia Florestal, UFPR, Curitiba, PR, Brasil - felipegust@ hotmail.com; danielepotulski@ufpr.br

${ }^{3}$ Arquiteta, M.Sc., Doutoranda em Engenharia Florestal, UFPR, Curitiba, PR, Brasil - janicebs@ gmail.com

${ }^{4}$ Eng. Químico, Mestrando em Engenharia Florestal, UFPR, Curitiba, PR, Brasil - mario.andrade@ momentive.com

${ }^{5}$ Eng ${ }^{\mathrm{a}}$ Florestal, Mestranda em Engenharia Florestal, UFPR, Curitiba, PR, Brasil - raquelmarchesan@ yahoo.com.br

Recebido para publicação: 03/02/2011 - Aceito para publicação: 02/01/2012

Esta pesquisa teve como objetivo avaliar a qualidade dos painéis compensados multilaminados produzidos a partir de lâminas de madeira de cinco espécies de pínus tropicais e nove espécies de eucalipto. Foram produzidos em laboratório painéis compensados com cinco lâminas de 2,0 mm de espessura, coladas com resina ureia-formaldeído (UF), com gramatura de $360 \mathrm{~g} / \mathrm{m}^{2}$. Os painéis foram prensados com pressão específica de $10 \mathrm{kgf} / \mathrm{cm}^{2}$, temperatura de $110^{\circ} \mathrm{C}$ e tempo de prensagem de 7 min. Foram avaliadas as propriedades de resistência da linha de cola aos esforços de cisalhamento (RLC), teste seco e úmido e flexão estática (MOE e MOR) paralela e perpendicular. Entre as espécies de pínus tropicais, o $P$. oocarpa e $P$. tecunumannii foram as que apresentaram melhor desempenho. Já para as espécies de eucalipto, os melhores resultados foram obtidos para E. saligna, E. viminalis e $E$. dunnii. Todas as espécies de pínus tropicais e de eucalipto avaliadas neste estudo apresentaram resultados de propriedades mecânicas estatisticamente iguais ou superiores em relação à espécie referencial - Pinus taeda. Os resultados obtidos indicam a viabilidade de uso de lâminas dessas espécies para fabricação de painéis compensados para uso interior.

Palavras-chave: Lâminas de madeira; compensados; pínus tropicais; eucalipto.

\begin{abstract}
Evaluation of potential use of species of tropical pine and eucalyptus for UF plywood manufacture. This research was developed in order to evaluate the quality of plywood manufactured from veneers of five species of tropical pine and nine species of eucalyptus. The plywood was manufactured with five veneers of $2.0 \mathrm{~mm}$ thickness, using urea-formaldehyde resin with amount of glue spread of $360 \mathrm{~g} / \mathrm{m}^{2}$. The boards were pressed at pressure of $10 \mathrm{kgf} / \mathrm{cm}^{2}$, temperatures of $110{ }^{\circ} \mathrm{C}$ and pressing time of 8 minutes. It was evaluated the properties of glue line shear strength (RLC) and static bending (MOE and MOR), parallel and perpendicular. Among tropical pine species, $P$. oocarpa and $P$. tecunumannii presented the best results in relation to mechanical properties of the boards. For the eucalyptus species best results were obtained by E. saligna, E. viminalis and E. dunnii. All the tropical pine and eucalyptus species evaluated in this research presented boards mechanical properties statistically equal or greater when compared to referential species - Pinus taeda. The results revealed the feasible use of such species veneers for plywood manufactures to internal applications.
\end{abstract}

Keywords: Veneers; plywood; tropical pine; eucalyptus.

\section{INTRODUÇÃO}

A contribuição econômica, social e ambiental do setor de florestas plantadas no Brasil é altamente significativa. Segundo dados de 2008, existem no Brasil 6,5 milhões de hectares de florestas plantadas, dos quais 4,26 milhões são plantios de eucalipto e 1,87 milhões de pínus. O setor contribui com arrecadações de impostos na ordem de R 9 bilhões e são gerados 2,2 milhões de empregos diretos e indiretos. $\mathrm{Na}$ área ambiental, sete a dez toneladas de $\mathrm{CO}_{2}$ são sequestrados por hectare/ano (SBS, 2008). 
No que se refere ao setor de indústrias de base florestal, a sua contribuição atinge níveis que correspondem a quase 3,5\% do PIB nacional, 8,5 milhões de empregos gerados e US\$ 8,5 bilhões em exportações, o que corresponde a 6,2\% do total de exportação brasileira (ASSOCIAÇÃO BRASILEIRA DA INDÚSTRIA DA MADEIRA PROCESSADA MECANICAMENTE (ABIMCI), 2007). Com relação à produção de painéis compensados, segundo dados do SBS (2008), a produção brasileira em 2007 foi cerca de 2,67 milhões de $\mathrm{m}^{3}$, sendo que, desse total, 1,98 milhões de $\mathrm{m}^{3}$ são produzidos com espécies de florestas plantadas (Pinus spp.) e 0,69 milhões de $\mathrm{m}^{3}$ com espécies de florestas nativas (madeiras tropicais).

Os dados demonstram que cerca de $70 \%$ de painéis compensados são produzidos com madeira de pínus provenientes de plantios florestais. A exploração extensiva das plantações de espécies tradicionais de Pinus (Pinus taeda e Pinus elliottii) tem gerado problemas de oferta de madeiras provenientes de árvores de grandes diâmetros para obtenção de lâminas de boa qualidade para produção de compensados. Vários estudos têm sido realizados em busca de espécies alternativas para produção de lâminas e compensados. Iwakiri et al. (2001) avaliaram a qualidade de painéis compensados produzidos com cinco espécies de pínus tropicais com 10 anos de idade e concluíram que as mesmas apresentam grande potencial para essa finalidade de uso. Keinert Jr. e Interanmense (1994) estudaram o comportamento de seis espécies do gênero Eucalyptus na produção de lâminas e compensados e obtiveram bons resultados nas avaliações qualitativas do material. Pio (1996) e Interanmense (1998) também estudaram o comportamento de quatro espécies de eucalipto para produção de painéis compensados para uso interior, obtendo resultados promissores quanto às suas propriedades mecânicas.

$\mathrm{Na}$ produção de compensados, os fatores relacionados à tecnologia devem ser analisados com muita atenção, principalmente quanto ao processo físico-químico envolvido na colagem de lâminas de madeira (MARRA, 1992). O tipo de resina empregado na colagem deve ser adequado ao ambiente de utilização do painel, podendo ser ureia-formaldeído para ambiente interno e fenol-formaldeído para ambiente externo. A formulação da batida de cola e a gramatura são parâmetros que irão influenciar diretamente na qualidade da colagem e ao mesmo tempo no custo de produção do compensado (BALDWIN, 1993; SELLERS, 1993). Os autores afirmam que a densidade da madeira é um fator importante na definição desses parâmetros, tendo em vista as interações que ocorrem entre a porosidade da madeira e a absorção do adesivo na formação da ligação adesiva entre as lâminas. De acordo com Marra (1992), madeiras de baixa densidade absorvem maior quantidade de adesivo devido a sua maior porosidade. Portanto, a viscosidade do adesivo deve ser aumentada, para evitar a formação da linha de cola "faminta". Outros fatores como pH e extrativos presentes na madeira são também importantes, podendo interferir na cura do adesivo durante o processo de prensagem do painel na prensa quente. Baldwin (1993) afirma que alguns extrativos presentes nas lâminas podem dificultar o processo de vaporização e sua migração de uma linha de cola para outra, e destas para as bordas do painel, para liberação ao ambiente externo. Esse processo, sendo muito lento, terá como consequência o aumento da pressão interna de vapor, resultando no "estouro" no momento da abertura da prensa e "delaminação" do painel. Portanto, a heterogeneidade e a variabilidade das propriedades anatômicas, físicas e químicas da madeira, entre as espécies de madeira, ou até mesmo entre as diferentes partes de uma árvore da mesma espécie, podem afetar as condições de colagem de lâminas durante o processo de fabricação de painéis compensados.

Esse trabalho teve como objetivo avaliar a qualidade de painéis compensados produzidos com lâminas de madeira de cinco espécies de pínus tropicais e nove espécies de eucalipto para produção de painéis compensados para uso interior.

\section{MATERIAL E MÉTODOS}

Foram objetos do presente estudo cinco espécies de pínus tropicais e nove espécies de eucalipto, com idade média na faixa de 20 a 25 anos, provenientes de florestas plantadas. As madeiras de Eucalyptus grandis, Eucalyptus saligna, Pinus taeda, Pinus oocarpa, Pinus caribaea, Pinus maximinoi, Pinus tecunumannii e Pinus chiapensis foram coletadas nos plantios florestais localizados na região de Ibaiti, PR. Já as madeiras de Eucalyptus dunnii, Eucalyptus globulus, Eucalyptus viminalis, Eucalyptus robusta, Eucalyptus phaeotricha, Eucalyptus deanei e Eucalyptus pellita foram coletadas nos plantios florestais localizados na região de Piên, PR, e Jaraguá do Sul, SC. O Pinus taeda foi utilizado como espécie de referência para efeitos de comparações de resultados, sendo que os painéis foram produzidos nas mesmas condições experimentais para todas as espécies em estudo. Para a colagem de lâminas, foi 
utilizada a resina ureia-formaldeído (UF) com teor de sólidos de $65 \%$, pH de 8,0 e viscosidade Brookfield de $420 \mathrm{cP}$. Sal de sulfato de amônia foi utilizado como catalisador.

As lâminas foram obtidas num torno-piloto, com espessura de $2,0 \mathrm{~mm}$, e foram seccionadas numa guilhotina pneumática com as dimensões de $600 \mathrm{~mm}$ x $600 \mathrm{~mm}$. A densidade básica da madeira foi determinada em amostras retiradas de lâminas de cada espécie, com dimensões de $20 \mathrm{~mm}$ x $300 \mathrm{~mm}$. Para a produção de painéis compensados, as lâminas foram secas em estufa ao teor de umidade médio de $8 \%$ e esquadrejadas em dimensões finais de $500 \mathrm{~mm}$ x $500 \mathrm{~mm}$.

Os painéis foram produzidos com cinco lâminas utilizando a resina uréia-formaldeído com a seguinte formulação em partes por peso: 100 partes de resina, 25 partes de farinha de trigo, 20 partes de água e 7 partes de catalisador. O adesivo, em quantidade de $360 \mathrm{~g} / \mathrm{m}^{2}$ (linha dupla), foi aplicado manualmente, com uma espátula, sobre a superfície da lâmina. Os painéis foram prensados com a pressão específica de $10 \mathrm{kgf} / \mathrm{cm}^{2}$, temperatura de $110^{\circ} \mathrm{C}$ e tempo de prensagem de 7 minutos. Os parâmetros do processo de manufatura dos painéis em laboratório foram definidos com base nas informações técnicas da literatura, fabricantes de resina e indústrias de compensados. Foram produzidos dois painéis por espécie, perfazendo um total de 30 painéis experimentais.

Após o processo de acondicionamento dos painéis na câmara climática com temperatura de $20 \pm$ $2{ }^{\circ} \mathrm{C}$ e umidade relativa de $65 \pm 5 \%$, foram retirados corpos de prova para realização dos seguintes ensaios em laboratório: resistência da linha de cola aos esforços de cisalhamento (teste seco e úmido) e flexão estática para determinação de módulo de elasticidade (MOE) e módulo de ruptura (MOR) no sentido paralelo e perpendicular. Os ensaios foram realizados de acordo com os procedimentos descritos na Norma Europeia EN 314 (1993) e EN 310 (1993), respectivamente. Os resultados dos ensaios foram analisados através de análise de variância e teste de Tukey ao nível de probabilidade de $95 \%$. O delineamento experimental foi inteiramente casualizado.

\section{RESULTADOS E DISCUSSÃO}

\section{Densidade básica das lâminas}

Os valores médios de densidade básica das lâminas das espécies estudadas estão apresentados na tabela 1.

Tabela 1. Densidade básica das lâminas das espécies estudadas.

Table 1. Veneers density of wood species.

\begin{tabular}{lc}
\hline Espécie & Densidade básica $\left(\mathbf{g} / \mathbf{c m}^{\mathbf{3}}\right)$ \\
\hline Pinus taeda & 0,571 \\
Pinus oocarpa & 0,556 \\
Pinus caribaea hondurensis & 0,465 \\
Pinus chiapensis & 0,394 \\
Pinus maximinoi & 0,413 \\
Pinus tecunumanii & 0,547 \\
Eucalyptus grandis & 0,436 \\
Eucalyptus saligna & 0,687 \\
Eucalyptus globulus & 0,564 \\
Eucalyptus viminalis & 0,617 \\
Eucalyptus dunnii & 0,561 \\
Eucalyptus robusta & 0,577 \\
Eucalyptus phaeotricha & 0,584 \\
Eucalyptus deanei & 0,571 \\
Eucalyptus pellita & 0,597 \\
\hline
\end{tabular}

Os valores médios de densidade básica das lâminas das espécies de pínus tropicais variaram na faixa de $0,394 \mathrm{~g} / \mathrm{cm}^{3}$ para Pinus chiapensis e $0,556 \mathrm{~g} / \mathrm{cm}^{3}$ para Pinus oocarpa. Para as lâminas de eucalipto, as densidades médias variaram de $0,436 \mathrm{~g} / \mathrm{cm}^{3}$ para Eucalyptus grandis e $0,687 \mathrm{~g} / \mathrm{cm}^{3}$ para Eucalyptus saligna. 
Os valores obtidos tanto para as espécies de pínus tropicais quanto para as de eucalipto estão dentro das faixas de valores de densidade apresentadas na literatura. Bortoletto Jr. (2003) obteve para 11 espécies de eucalipto os seguintes valores médios de densidade básica: E. pilulararis $-0,67 \mathrm{~g} / \mathrm{cm}^{3}, E$. propinqua $-0,77 \mathrm{~g} / \mathrm{cm}^{3}$, E. microcorys $-0,63 \mathrm{~g} / \mathrm{cm}^{3}$, E. maculata $-0,71 \mathrm{~g} / \mathrm{cm}^{3}$, E. pyrocarpa $-0,64 \mathrm{~g} / \mathrm{cm}^{3}$, E. tereticornis - $0,74 \mathrm{~g} / \mathrm{cm}^{3}$, E. urophylla $-0,60 \mathrm{~g} / \mathrm{cm}^{3}$, E. pellita $-0,65 \mathrm{~g} / \mathrm{cm}^{3}$, E. citriodora $-0,76 \mathrm{~g} / \mathrm{cm}^{3}$, E. torelliana - 0,61 g/ $\mathrm{cm}^{3}$ e E. saligna $-0,56 \mathrm{~g} / \mathrm{cm}^{3}$. Interanmense (1998) encontrou, para lâminas de Eucalyptus cloeziana, Eucalyptus punctata e Eucalyptus maculata, valores médios de densidade básica de 0.70, 0.76 e $0.78 \mathrm{~g} / \mathrm{cm}^{3}$, respectivamente. Já Pio (1996) obteve para lâminas de Eucalyptus scabra e Eucalyptus robusta densidade básica de 0,64 e $0,56 \mathrm{~g} / \mathrm{cm}^{3}$, respectivamente.

Trianoski et al. (2008) encontraram, para espécies de pínus tropicais, os seguintes valores de densidade básica: $P$. tecunumanii - $0,489 \mathrm{~g} / \mathrm{cm}^{3}, P$. oocarpa $-0,481 \mathrm{~g} / \mathrm{cm}^{3}, P$. taeda $-0,471 \mathrm{~g} / \mathrm{cm}^{3}, P$. maximinoi - $0,451 \mathrm{~g} / \mathrm{cm}^{3}, P$. caribaea bahamensis - $0,429 \mathrm{~g} / \mathrm{cm}^{3}, P$. caribaea hondurensis $-0,424 \mathrm{~g} / \mathrm{cm}^{3}$, P. caribaea caribaea $-0,398 \mathrm{~g} / \mathrm{cm}^{3}$ e $P$. chiapensis - $0,395 \mathrm{~g} / \mathrm{cm}^{3}$.

\section{Cisalhamento da linha de cola}

Os resultados de ensaios de cisalhamento da linha de cola no teste seco e úmido estão apresentados na tabela 2.

Os valores médios de tensões de cisalhamento da linha de cola variaram na faixa de 1,48 $\mathrm{MPa}$ (E. deanei) a 2,61 MPa (P. oocarpa) para o teste seco, e de $0,85 \mathrm{MPa}$ (P. caribaea hondurensis) a 2,39 MPa (E. phaeotricha) para o teste úmido.

Tabela 2. Resultados de cisalhamento da linha de cola.

Table 2. Results of glue line shear strength.

\begin{tabular}{lcccc}
\hline \multirow{2}{*}{ Espécie } & \multicolumn{2}{c}{ Teste seco (MPa) } & \multicolumn{2}{c}{ Teste úmido $(\mathbf{M P a})$} \\
\cline { 2 - 5 } & Média $(\mathbf{C V})$ & FM $(\%)$ & Média $(\mathbf{C V})$ & FM $(\%)$ \\
\hline Pinus taeda & $1,95 \mathrm{bcd}(17,41)$ & 24 & $1,17 \mathrm{de}(25,64)$ & 23 \\
Pinus oocarpa & $2,61 \mathrm{a}(9,36)$ & 84 & $1,59 \mathrm{bcd}(10,82)$ & 18 \\
Pinus caribaea hondurensis & $1,55 \mathrm{~cd}(12,63)$ & 64 & $0,85 \mathrm{e}(9,96)$ & 3 \\
Pinus chiapensis & $1,84 \mathrm{bcd}(6,00)$ & 61 & $1,18 \mathrm{cde}(16,66)$ & 4 \\
Pinus maximinoi & $2,04 \mathrm{bc}(9,76)$ & 91 & $1,38 \mathrm{cde}(29,72)$ & 41 \\
Pinus tecunumanii & $1,87 \mathrm{bcd}(14,72)$ & 12 & $1,26 \mathrm{cde}(17,94)$ & 7 \\
Eucalyptus grandis & $1,79 \mathrm{bcd}(9,47)$ & 64 & $1,25 \mathrm{cde}(12,98)$ & 45 \\
Eucalyptus saligna & $2,13 \mathrm{abc}(24,61)$ & 22 & $1,67 \mathrm{bcd}(22,25)$ & 5 \\
Eucalyptus globulus & $1,94 \mathrm{bcd}(17,78)$ & 59 & $1,63 \mathrm{bcd}(28,71)$ & 46 \\
Eucalyptus viminalis & $2,27 \mathrm{ab}(22,01)$ & 54 & $1,71 \mathrm{bc}(40,19)$ & 63 \\
Eucalyptus dunnii & $2,14 \mathrm{ab}(10,49)$ & 38 & $2,09 \mathrm{abc}(14,62)$ & 86 \\
Eucalyptus robusta & $2,18 \mathrm{ab}(17,96)$ & 67 & $2,04 \mathrm{ab}(27,49)$ & 33 \\
Eucalyptus phaeotricha & $2,13 \mathrm{ab}(11,80)$ & 82 & $2,39 \mathrm{a}(13,36)$ & 77 \\
Eucalyptus deanei & $1,48 \mathrm{~d}(15,92)$ & 82 & $1,34 \mathrm{cde}(14,45)$ & 44 \\
Eucalyptus pellita & $2,09 \mathrm{~b}(19,52)$ & 74 & $2,04 \mathrm{ab}(10,97)$ & 67 \\
\hline
\end{tabular}

CV: coeficiente de variação; FM: percentagem de falhas na madeira. Médias seguidas de mesmas letras na coluna são estatisticamente iguais ao nível de probabilidade de $95 \%$.

Para o teste seco, os painéis de $P$. oocarpa apresentaram melhor desempenho, tendo sua média estatisticamente igual em relação aos painéis de E. saligna, E. viminalis, E. dunnii, E. robusta e E. phaeotricha, e estatisticamente superior em relação às demais espécies.

Com relação ao teste úmido, os painéis de E. phaeotricha apresentaram média estatisticamente igual em relação aos painéis de E. pellita, E. robusta e E. dunnii, e superior em relação às demais espécies.

Tanto para o teste seco quanto para o teste úmido, os painéis de todas as espécies de pínus tropicais e de eucalipto apresentaram valores médios de tensões de cisalhamento estaticamente iguais ou superiores em comparação aos painéis de $P$. taeda, utilizada como espécie referencial, tendo como destaque o P. oocarpa. 
A avaliação de percentagens de falhas na madeira demonstrou que, com exceção de $P$. tecunumanii, E. saligna e E. dunnii, todas as demais espécies de pínus tropicais e eucalipto apresentaram percentual acima de $60 \%$.

Os valores médios de tensões de cisalhamento da linha de cola obtidos para as cinco espécies de pínus tropicais e nove espécies de eucalipto estão dentro da faixa de valores apresentados na literatura. Iwakiri et al. (2002) encontraram para painéis de $P$. taeda e $P$. oocarpa, produzidos com resina UF, valores médios de tensões de cisalhamento (teste seco) de 1,57 MPa e 1,81 MPa, respectivamente. Num outro estudo realizado por Iwakiri et al. (2001) para painéis compensados ureicos de $P$. caribaea, $P$. chiapensis, $P$. maximinoi, $P$. oocarpa e $P$. tecunumanii, foram obtidos valores médios de tensões de cisalhamento na faixa de 1,21 MPa a 1,62 MPa para teste seco, e de 0,55 MPa a 1,31 MPa para teste úmido.

Para compensados de eucalipto, Pio (1996) obteve, para E. scabra e E. robusta, resistência da linha de cola no teste seco de 2,36 MPa e 2,57 MPa, e no teste úmido de 2,31 MPa e 2,32 $\mathrm{MPa}$, respectivamente para as duas espécies. Interanmense (1998), estudando as espécies de E. cloeziana e $E$. maculata, encontrou para o teste seco valores médios de resistência da linha de cola de 3,25 MPa e 2,81 MPa, e, para o teste úmido, valores médios de 2,47 MPa e 2,57 MPa, respectivamente para as duas espécies.

Tanto no teste seco quanto no teste úmido, com exceção de Pinus caribaea hondurensis, todas as demais espécies estudadas apresentaram valores médios de tensões de cisalhamento superiores ao valor mínimo de 1,0 MPa, requerido pela norma EN 314-2:1993.

\section{Flexão estática paralela}

Na tabela 3 estão apresentados os valores médios e coeficientes de variação para Módulo de Elasticidade (MOE) e Módulo de Ruptura (MOR) na direção paralela.

Tabela 3. Flexão estática paralela.

Table 3. Static bending - parallel direction.

\begin{tabular}{lcccc}
\hline \multirow{2}{*}{ Espécie } & \multicolumn{2}{c}{ MOE (MPa) } & \multicolumn{2}{c}{ MOR (MPa) } \\
\cline { 2 - 5 } & Média & CV $(\%)$ & Média & CV $(\%)$ \\
\hline Pinus taeda & $8.541 \mathrm{de}$ & 14,07 & $72,96 \mathrm{de}$ & 12,10 \\
Pinus oocarpa & $11.653 \mathrm{bc}$ & 24,35 & $92,27 \mathrm{abcd}$ & 24,57 \\
Pinus caribaea hondurensis & $6.300 \mathrm{e}$ & 15,05 & $61,72 \mathrm{e}$ & 14,25 \\
Pinus chiapensis & $7.370 \mathrm{e}$ & 7,32 & $58,54 \mathrm{e}$ & 9,79 \\
Pinus maximinoi & $7.233 \mathrm{e}$ & 13,11 & $60,55 \mathrm{e}$ & 11,48 \\
Pinus tecunumanii & $13.714 \mathrm{ab}$ & 13,27 & $101,83 \mathrm{ab}$ & 15,29 \\
Eucalyptus grandis & $11.618 \mathrm{bc}$ & 10,04 & $94,44 \mathrm{abcd}$ & 13,99 \\
Eucalyptus saligna & $15.532 \mathrm{a}$ & 11,91 & $104,54 \mathrm{a}$ & 15,68 \\
Eucalyptus globulus & $11.119 \mathrm{bcd}$ & 14,02 & $76,64 \mathrm{cde}$ & 15,82 \\
Eucalyptus viminalis & $16.366 \mathrm{a}$ & 3,59 & $94,91 \mathrm{abcd}$ & 13,03 \\
Eucalyptus dunnii & $16.319 \mathrm{a}$ & 5,40 & $101,54 \mathrm{ab}$ & 6,80 \\
Eucalyptus robusta & $11.464 \mathrm{bc}$ & 4,25 & $85,78 \mathrm{abcd}$ & 7,89 \\
Eucalyptus phaeotricha & $12.747 \mathrm{bc}$ & 7,31 & $99,35 \mathrm{abc}$ & 7,65 \\
Eucalyptus deanei & $10.248 \mathrm{~cd}$ & 13,22 & $75,19 \mathrm{de}$ & 5,38 \\
Eucalyptus pellita & $11.894 \mathrm{bc}$ & 8,93 & $81,08 \mathrm{bcde}$ & 12,86 \\
\hline CV
\end{tabular}

CV: coeficiente de variação. Médias seguidas de mesmas letras na coluna são estatisticamente iguais ao nível de probabilidade de $95 \%$.

A amplitude de variação dos valores médios de MOE paralelo foi de $6.300 \mathrm{MPa}(P$. caribaea hondurensis) a $16.366 \mathrm{MPa}$ (E. viminalis). Para o MOR paralelo, a variação foi de 58,54 MPa ( $P$. chiapensis) a 104,54 MPa (E. saligna).

Os painéis de E. viminalis, E. dunnii e E. saligna apresentaram valores médios de MOE paralelo estatisticamente iguais em relação aos painéis de $P$. tecunumanii e superiores em relação aos painéis das demais espécies. Para o MOR paralelo, os painéis de E. saligna apresentaram média estatisticamente iguais em relação aos painéis de $P$. tecunumanii, E. dunnii, E. phaeotricha, E. viminalis, $P$. oocarpa e E. robusta, e superior em relação aos painéis das demais espécies. 
Os painéis de P. oocarpa, P. tecunumanii, E. grandis, E. saligna, E. globulus, E. viminalis, E. dunnii, E. robusta, E. phaeotricha e E. pellita apresentaram valores médios de MOE paralelo estatisticamente superiores em relação à espécie referencial $-P$. taeda. Para o MOR paralelo, os painéis de $P$. tecunumanii, E. saligna, E. dunnii e E. phaeotricha foram os que apresentaram médias estatisticamente superiores em relação à espécie referencial.

Tanto para o MOE, quanto para o MOR paralelo, todas as espécies de pínus tropicais e de eucalipto apresentaram valores médios estatisticamente iguais ou superiores em relação à espécie referencial - Pinus taeda. A densidade das lâminas não influenciou claramente nos resultados de MOE e MOR paralelo.

Com relação aos trabalhos apresentados na literatura, Iwakiri et al. (2002) encontraram, para painéis de $P$. taeda e $P$. oocarpa produzidos com resina ureia-formaldeído, valores médios de MOE paralelo de $9.486 \mathrm{MPa}$ e $10.467 \mathrm{MPa}$, respectivamente. Para o MOR paralelo, os autores encontraram valores médios de 73,29 MPa e 75,55 MPa, respectivamente para as duas espécies de pínus. Num outro trabalho realizado por Iwakiri et al. (2001), os autores encontraram, para cinco espécies de pínus tropicais com 10 anos de idade, valores médios de MOE paralelo variando na faixa de $3.877 \mathrm{MPa}$ a $6.838 \mathrm{MPa}$. Para o MOR paralelo, os valores obtidos variaram de 33,11 MPa a 39,49 MPa. Portanto, pode-se afirmar que as espécies estudadas neste trabalho apresentaram desempenho satisfatório quanto às propriedades de MOE e MOR paralelo.

Para compensados de eucalipto, Keinert Jr. e Interanmense (1994) obtiveram, para E. dunnii, E. robusta, E. viminalis, E. grandis e E. saligna, valores médios de MOE paralelo de 12.123, 12.152, $13.279,14.024$ e $11.539 \mathrm{MPa}$, respectivamente. Já para o MOR paralelo, os valores médios encontrados foram respectivamente de 53.7, 75.9, 60.1, 58.2 e 54.0 MPa.

\section{Flexão estática perpendicular}

Na tabela 4 estão apresentados os valores médios e coeficientes de variação para Módulo de Elasticidade (MOE) e Módulo de Ruptura (MOR) na direção perpendicular.

Tabela 4. Flexão estática perpendicular.

Table 4. Static bending - cross direction.

\begin{tabular}{lcccc}
\hline \multirow{2}{*}{ Espécie } & \multicolumn{2}{c}{ MOE (MPa) } & \multicolumn{2}{c}{ MOR (MPa) } \\
\cline { 2 - 5 } & Média & CV (\%) & Média & CV (\%) \\
\hline Pinus taeda & $2.655 \mathrm{cdefg}$ & 12,51 & $32,44 \mathrm{~cd}$ & 11,74 \\
Pinus oocarpa & $3.841 \mathrm{ab}$ & 12,51 & $45,24 \mathrm{ab}$ & 14,51 \\
Pinus caribaea hondurensis & $2.046 \mathrm{~g}$ & 18,56 & $28,25 \mathrm{~d}$ & 14,36 \\
Pinus chiapensis & $2.503 \mathrm{efg}$ & 7,22 & $29,23 \mathrm{~d}$ & 12,48 \\
Pinus maximinoi & $2.223 \mathrm{fg}$ & 12,09 & $32,75 \mathrm{~cd}$ & 6,66 \\
Pinus tecunumanii & $3.347 \mathrm{abcde}$ & 23,87 & $41,93 \mathrm{abc}$ & 10,60 \\
Eucalyptus grandis & $3.524 \mathrm{abc}$ & 6,43 & $38,17 \mathrm{bcd}$ & 15,64 \\
Eucalyptus saligna & $4.062 \mathrm{a}$ & 7,12 & $52,20 \mathrm{a}$ & 8,87 \\
Eucalyptus globulus & $3.130 \mathrm{bcde}$ & 21,51 & $38,17 \mathrm{bcd}$ & 20,03 \\
Eucalyptus viminalis & $3.884 \mathrm{ab}$ & 10,15 & $41,38 \mathrm{abc}$ & 12,27 \\
Eucalyptus dunnii & $3.930 \mathrm{ab}$ & 12,61 & $43,01 \mathrm{abc}$ & 9,26 \\
Eucalyptus robusta & $3.070 \mathrm{bcdef}$ & 11,72 & $40,98 \mathrm{abc}$ & 18,28 \\
Eucalyptus phaeotricha & $3.746 \mathrm{ab}$ & 8,44 & $50,01 \mathrm{a}$ & 8,34 \\
Eucalyptus deanei & $2.522 \mathrm{defg}$ & 11,58 & $33,43 \mathrm{~cd}$ & 10,95 \\
Eucalyptus pellita & $3.400 \mathrm{abcd}$ & 16,40 & $42,16 \mathrm{abc}$ & 25,32 \\
\hline CV:
\end{tabular}

CV: coeficiente de variação. Médias seguidas de mesmas letras na coluna são estatisticamente iguais ao nível de probabilidade de $95 \%$.

A amplitude de variação dos valores médios de MOE perpendicular foi de $2.046 \mathrm{MPa}(P$. caribaea hondurensis) a $4.062 \mathrm{MPa}$ (E. saligna). Para o MOR perpendicular, a variação foi de $28,25 \mathrm{MPa}$ (P. caribaea hondurensis) a 52,20 MPa (E. saligna).

Os painéis de E. saligna apresentaram valores médios de MOE perpendicular estatisticamente iguais em relação aos painéis de $P$. oocarpa, P. tecunumanii, E. grandis, E. viminalis, E. dunnii, E. phaeotricha e E. pellita, e superiores em relação aos painéis das demais espécies. Para o MOR 
perpendicular, os painéis de E. saligna e E. phaeotricha apresentaram média estatisticamente igual em relação aos painéis de $P$. oocarpa, P. tecunumanii, E. viminalis, E. dunnii, E. robusta, e E. pellita, e superiores em relação aos painéis das demais espécies.

Não foram encontrados na literatura resultados de MOE e MOR perpendicular para as espécies de pínus tropicais, entretanto, para compensados de eucalipto, Keinert Jr. e Interanmense (1994) obtiveram, para E.dunnii, E. robusta, E. viminalis, E. grandis e E. saligna, valores médios de MOE perpendicular de 3.286, 3.045, 2.503, 3.833 e $4.253 \mathrm{MPa}$, respectivamente para as espécies estudadas. Já para o MOR perpendicular, os valores médios encontrados foram, respectivamente, de 37.6, 32.4, 22.2, 30.7 e $40.7 \mathrm{MPa}$.

Tanto para o MOE quanto para o MOR perpendicular, todas as espécies de pínus tropicais e de eucalipto apresentaram valores médios estatisticamente iguais ou superiores em relação à espécie referencial - Pinus taeda. A densidade das lâminas não influenciou claramente nos resultados de MOE e MOR perpendicular.

\section{CONCLUSÕES}

Com base nos resultados obtidos nesta pesquisa, as seguintes conclusões podem ser apresentadas:

- As densidades básicas das lâminas das espécies estudadas não influenciaram diretamente nos resultados de MOE e MOR paralelo e perpendicular dos painéis;

- Nos ensaios de cisalhamento da linha de cola, os painéis de P. oocarpa, E. saligna, E. viminalis, E. dunnii, E. robusta e E. phaeotricha apresentaram melhor desempenho no teste seco. Para o teste úmido, os painéis de E. phaeotricha, E. pellita, E. robusta e E. dunnii foram os que apresentaram melhores resultados.

- Nos ensaios de flexão estática paralela, os painéis de E. viminalis, E. dunnii e E. saligna apresentaram maiores valores médios de MOE. Os painéis de E. saligna, P. tecunumanii, E. dunnii, E. phaeotricha, E. viminalis, $P$. oocarpa e E. robusta foram as que apresentaram melhores resultados de MOR.

- Nos ensaios de flexão estática perpendicular, os painéis de E. saligna, E. dunnii, E. viminalis, P. oocarpa e E. phaeotricha foram as espécies que apresentaram melhores resultados de MOE. Os painéis de E. saligna, E. phaeotricha e $P$. oocarpa foram os que apresentaram melhores resultados de MOR.

- Nas avaliações gerais dos resultados, o $P$. tecunumanii e $P$. oocarpa foram as espécies de pínus tropicais com melhor desempenho. Já para as espécies de eucalipto, os melhores resultados foram obtidos para E. saligna, E. viminalis e E. dunnii.

- Todas as espécies de pínus tropicais e de eucalipto avaliadas neste estudo apresentaram resultados de cisalhamento da linha de cola e de flexão estática estatisticamente iguais ou superiores em relação à espécie referencial-Pinus taeda.

\section{REFERÊNCIAS}

ASSOCIAÇÃO BRASILEIRA DA INDÚSTRIA DA MADEIRA PROCESSADA MECANICAMENTE (ABIMCI). Estudo Setorial. Curitiba, 2007, 40 p.

BALDWIN, R. F. Plywood and veneer-based products: manufacturing practices. San Francisco: Miller Freeman, 1993. 388 p.

BORTOLETTO JR., G. Produção de compensados com 11 espécies do gênero Eucalyptus, avaliação das suas propriedades físico-mecânicas e indicações para utilização. Scientia Forestalis, v. 63, p. 65 - 78, jun. 2003.

EUROPEAN COMMITTEE FOR STANDARDIZATION. EN 310: 1993: Plywood - Determination of modulus of elasticity and modulus of rupture in static bending. 1993.

EN 314-2: 1993: Plywood - Determination of shear bonding strength. 1993. 
INTERANMENSE, M. T. Utilização das madeiras de Eucalyptus cloeziana (F. Muell), Eucalyptus maculata (Hook) e Eucalyptus punctata DC var. punctata para produção de painéis compensados. Dissertação (Mestrado, Curso de Pós-Graduação em Engenharia Florestal) - Universidade Federal do Paraná, 1998, 81 p.

IWAKIRI, S.; OLANDOSKI, D. P.; LEONHARDT, G.; BRAND, M. A. 2001. Produção de chapas de madeira compensada de cinco espécies de pínus tropicais. Ciência Florestal, Santa Maria, v. 11, n. 2, p. $71-77,2001$.

IWAKIRI, S.; SILVA, J. C.; SILVA, J. R. M.; ALVES, C. R.; PUEHRINGER, C. A. Produção de compensados de Pinus taeda L. e Pinus oocarpa Schiede com diferentes formulações de adesivo ureiaformaldeído. Árvore, v. 26, n. 3, p. 371 - 375, 2002.

KEINERT JR., S.; INTERANMENSE, M. T. Laminação, produção e testes de compensados a partir de Eucalyptus spp. Relatório de projeto de pesquisa apresentado ao CNPq. 94 p. Curitiba, 1994.

MARRA, A. A. 1992. Technology of wood bonding: principles in practice. New York: Van Nostrand Reinhold. 1992. 453 p.

PIO, N. S. Avaliação da madeira de Eucalyptus scabra (Dum-Cours) e Eucalyptus robusta (Smith) na produção de painéis compensados. Dissertação (Mestrado, Curso de Pós-Graduação em Engenharia Florestal) - Universidade Federal do Paraná, 1996, 101 p.

SBS, 2008. Fatos e números do Brasil Florestal - 2008. Disponível em: <www.sbs.org.br〉. Acesso em $31 / 01 / 2011$.

SELLERS, T. Plywood and adhesive technology. New York: Marcel Dekker, 1993. 661 p.

TRIANOSKI, R.; PRATA, J. G.; MATOS, J. L. M. Variação da massa específica básica em sete espécies de pínus tropicais. I SIMADERJ, Simpósio de Ciência e Tecnologia da Madeira do Estado do Rio de Janeiro. Anais... Seropédica, RJ, 2008. (sem paginação). 